\title{
Miscellaneous Facts about Open Functions and Continuous Functions
}

\author{
Artur Korniłowicz \\ Institute of Informatics \\ University of Białystok \\ Sosnowa 64, 15-887 Białystok, Poland
}

\begin{abstract}
Summary. In this article we give definitions of open functions and continuous functions formulated in terms of "balls" of given topological spaces.
\end{abstract}

MML identifier: TOPS_4, version: $\underline{7.11 .07 \quad 4.156 .1112}$

The notation and terminology used here have been introduced in the following papers: [6], [4], [5], [8], [1], [2], [3], [10], [11], [12], [7], [9], and [13].

\section{Open FunCtions}

We adopt the following rules: $n, m$ are elements of $\mathbb{N}, T$ is a non empty topological space, and $M, M_{1}, M_{2}$ are non empty metric spaces.

The following propositions are true:

(1) Let $A, B, S, T$ be topological spaces, $f$ be a function from $A$ into $S$, and $g$ be a function from $B$ into $T$. Suppose that

(i) the topological structure of $A=$ the topological structure of $B$,

(ii) the topological structure of $S=$ the topological structure of $T$,

(iii) $f=g$, and

(iv) $f$ is open.

Then $g$ is open.

(2) Let $P$ be a subset of $\mathcal{E}_{\mathrm{T}}^{m}$. Then $P$ is open if and only if for every point $p$ of $\mathcal{E}_{\mathrm{T}}^{m}$ such that $p \in P$ there exists a positive real number $r$ such that $\operatorname{Ball}(p, r) \subseteq P$. 
(3) Let $X, Y$ be non empty topological spaces and $f$ be a function from $X$ into $Y$. Then $f$ is open if and only if for every point $p$ of $X$ and for every open subset $V$ of $X$ such that $p \in V$ there exists an open subset $W$ of $Y$ such that $f(p) \in W$ and $W \subseteq f^{\circ} V$.

(4) Let $f$ be a function from $T$ into $M_{\text {top }}$. Then $f$ is open if and only if for every point $p$ of $T$ and for every open subset $V$ of $T$ and for every point $q$ of $M$ such that $q=f(p)$ and $p \in V$ there exists a positive real number $r$ such that $\operatorname{Ball}(q, r) \subseteq f^{\circ} V$.

(5) Let $f$ be a function from $M_{\text {top }}$ into $T$. Then $f$ is open if and only if for every point $p$ of $M$ and for every positive real number $r$ there exists an open subset $W$ of $T$ such that $f(p) \in W$ and $W \subseteq f^{\circ} \operatorname{Ball}(p, r)$.

(6) Let $f$ be a function from $\left(M_{1}\right)_{\text {top }}$ into $\left(M_{2}\right)_{\text {top }}$. Then $f$ is open if and only if for every point $p$ of $M_{1}$ and for every point $q$ of $M_{2}$ and for every positive real number $r$ such that $q=f(p)$ there exists a positive real number $s$ such that $\operatorname{Ball}(q, s) \subseteq f^{\circ} \operatorname{Ball}(p, r)$.

(7) Let $f$ be a function from $T$ into $\mathcal{E}_{\mathrm{T}}^{m}$. Then $f$ is open if and only if for every point $p$ of $T$ and for every open subset $V$ of $T$ such that $p \in V$ there exists a positive real number $r$ such that $\operatorname{Ball}(f(p), r) \subseteq f^{\circ} V$.

(8) Let $f$ be a function from $\mathcal{E}_{\mathrm{T}}^{m}$ into $T$. Then $f$ is open if and only if for every point $p$ of $\mathcal{E}_{\mathrm{T}}^{m}$ and for every positive real number $r$ there exists an open subset $W$ of $T$ such that $f(p) \in W$ and $W \subseteq f^{\circ} \operatorname{Ball}(p, r)$.

(9) Let $f$ be a function from $\mathcal{E}_{\mathrm{T}}^{m}$ into $\mathcal{E}_{\mathrm{T}}^{n}$. Then $f$ is open if and only if for every point $p$ of $\mathcal{E}_{\mathrm{T}}^{m}$ and for every positive real number $r$ there exists a positive real number $s$ such that $\operatorname{Ball}(f(p), s) \subseteq f^{\circ} \operatorname{Ball}(p, r)$.

(10) Let $f$ be a function from $T$ into $\mathbb{R}^{1}$. Then $f$ is open if and only if for every point $p$ of $T$ and for every open subset $V$ of $T$ such that $p \in V$ there exists a positive real number $r$ such that $] f(p)-r, f(p)+r\left[\subseteq f^{\circ} V\right.$.

(11) Let $f$ be a function from $\mathbb{R}^{1}$ into $T$. Then $f$ is open if and only if for every point $p$ of $\mathbb{R}^{\mathbf{1}}$ and for every positive real number $r$ there exists an open subset $V$ of $T$ such that $f(p) \in V$ and $\left.V \subseteq f^{\circ}\right] p-r, p+r[$.

(12) Let $f$ be a function from $\mathbb{R}^{\mathbf{1}}$ into $\mathbb{R}^{\mathbf{1}}$. Then $f$ is open if and only if for every point $p$ of $\mathbb{R}^{\mathbf{1}}$ and for every positive real number $r$ there exists a positive real number $s$ such that $] f(p)-s, f(p)+s\left[\subseteq f^{\circ}\right] p-r, p+r[$.

(13) Let $f$ be a function from $\mathcal{E}_{\mathrm{T}}^{m}$ into $\mathbb{R}^{1}$. Then $f$ is open if and only if for every point $p$ of $\mathcal{E}_{\mathrm{T}}^{m}$ and for every positive real number $r$ there exists a positive real number $s$ such that $] f(p)-s, f(p)+s\left[\subseteq f^{\circ} \operatorname{Ball}(p, r)\right.$.

(14) Let $f$ be a function from $\mathbb{R}^{1}$ into $\mathcal{E}_{\mathrm{T}}^{m}$. Then $f$ is open if and only if for every point $p$ of $\mathbb{R}^{\mathbf{1}}$ and for every positive real number $r$ there exists a positive real number $s$ such that $\left.\operatorname{Ball}(f(p), s) \subseteq f^{\circ}\right] p-r, p+r[$. 


\section{Continuous Functions}

Next we state a number of propositions:

(15) Let $f$ be a function from $T$ into $M_{\text {top }}$. Then $f$ is continuous if and only if for every point $p$ of $T$ and for every point $q$ of $M$ and for every positive real number $r$ such that $q=f(p)$ there exists an open subset $W$ of $T$ such that $p \in W$ and $f^{\circ} W \subseteq \operatorname{Ball}(q, r)$.

(16) Let $f$ be a function from $M_{\text {top }}$ into $T$. Then $f$ is continuous if and only if for every point $p$ of $M$ and for every open subset $V$ of $T$ such that $f(p) \in V$ there exists a positive real number $s$ such that $f^{\circ} \operatorname{Ball}(p, s) \subseteq V$.

(17) Let $f$ be a function from $\left(M_{1}\right)_{\text {top }}$ into $\left(M_{2}\right)_{\text {top }}$. Then $f$ is continuous if and only if for every point $p$ of $M_{1}$ and for every point $q$ of $M_{2}$ and for every positive real number $r$ such that $q=f(p)$ there exists a positive real number $s$ such that $f^{\circ} \operatorname{Ball}(p, s) \subseteq \operatorname{Ball}(q, r)$.

(18) Let $f$ be a function from $T$ into $\mathcal{E}_{\mathrm{T}}^{m}$. Then $f$ is continuous if and only if for every point $p$ of $T$ and for every positive real number $r$ there exists an open subset $W$ of $T$ such that $p \in W$ and $f^{\circ} W \subseteq \operatorname{Ball}(f(p), r)$.

(19) Let $f$ be a function from $\mathcal{E}_{\mathrm{T}}^{m}$ into $T$. Then $f$ is continuous if and only if for every point $p$ of $\mathcal{E}_{\mathrm{T}}^{m}$ and for every open subset $V$ of $T$ such that $f(p) \in V$ there exists a positive real number $s$ such that $f^{\circ} \operatorname{Ball}(p, s) \subseteq V$.

(20) Let $f$ be a function from $\mathcal{E}_{\mathrm{T}}^{m}$ into $\mathcal{E}_{\mathrm{T}}^{n}$. Then $f$ is continuous if and only if for every point $p$ of $\mathcal{E}_{\mathrm{T}}^{m}$ and for every positive real number $r$ there exists a positive real number $s$ such that $f^{\circ} \operatorname{Ball}(p, s) \subseteq \operatorname{Ball}(f(p), r)$.

(21) Let $f$ be a function from $T$ into $\mathbb{R}^{\mathbf{1}}$. Then $f$ is continuous if and only if for every point $p$ of $T$ and for every positive real number $r$ there exists an open subset $W$ of $T$ such that $p \in W$ and $\left.f^{\circ} W \subseteq\right] f(p)-r, f(p)+r[$.

(22) Let $f$ be a function from $\mathbb{R}^{\mathbf{1}}$ into $T$. Then $f$ is continuous if and only if for every point $p$ of $\mathbb{R}^{\mathbf{1}}$ and for every open subset $V$ of $T$ such that $f(p) \in V$ there exists a positive real number $s$ such that $\left.f^{\circ}\right] p-s, p+s[\subseteq V$.

(23) Let $f$ be a function from $\mathbb{R}^{1}$ into $\mathbb{R}^{1}$. Then $f$ is continuous if and only if for every point $p$ of $\mathbb{R}^{\mathbf{1}}$ and for every positive real number $r$ there exists a positive real number $s$ such that $\left.f^{\circ}\right] p-s, p+s[\subseteq] f(p)-r, f(p)+r[$.

(24) Let $f$ be a function from $\mathcal{E}_{\mathrm{T}}^{m}$ into $\mathbb{R}^{\mathbf{1}}$. Then $f$ is continuous if and only if for every point $p$ of $\mathcal{E}_{\mathrm{T}}^{m}$ and for every positive real number $r$ there exists a positive real number $s$ such that $\left.f^{\circ} \operatorname{Ball}(p, s) \subseteq\right] f(p)-r, f(p)+r[$.

(25) Let $f$ be a function from $\mathbb{R}^{\mathbf{1}}$ into $\mathcal{E}_{\mathrm{T}}^{m}$. Then $f$ is continuous if and only if for every point $p$ of $\mathbb{R}^{\mathbf{1}}$ and for every positive real number $r$ there exists a positive real number $s$ such that $\left.f^{\circ}\right] p-s, p+s[\subseteq \operatorname{Ball}(f(p), r)$. 


\section{REFERENCES}

[1] Grzegorz Bancerek. The fundamental properties of natural numbers. Formalized Mathematics, 1(1):41-46, 1990.

[2] Grzegorz Bancerek. The ordinal numbers. Formalized Mathematics, 1(1):91-96, 1990.

[3] Leszek Borys. Paracompact and metrizable spaces. Formalized Mathematics, 2(4):481485, 1991.

[4] Czesław Byliński. Functions and their basic properties. Formalized Mathematics, 1(1):55$65,1990$.

[5] Czesław Byliński. Functions from a set to a set. Formalized Mathematics, 1(1):153-164, 1990.

[6] Agata Darmochwał. The Euclidean space. Formalized Mathematics, 2(4):599-603, 1991.

[7] Agata Darmochwał and Yatsuka Nakamura. Metric spaces as topological spaces - fundamental concepts. Formalized Mathematics, 2(4):605-608, 1991.

[8] Stanisława Kanas, Adam Lecko, and Mariusz Startek. Metric spaces. Formalized Mathematics, 1(3):607-610, 1990.

[9] Artur Korniłowicz and Yasunari Shidama. Intersections of intervals and balls in $\mathcal{E}_{\mathrm{T}}^{n}$. Formalized Mathematics, 12(3):301-306, 2004.

[10] Beata Padlewska and Agata Darmochwał. Topological spaces and continuous functions. Formalized Mathematics, 1(1):223-230, 1990.

[11] Konrad Raczkowski and Paweł Sadowski. Topological properties of subsets in real numbers. Formalized Mathematics, 1(4):777-780, 1990.

[12] Edmund Woronowicz. Relations defined on sets. Formalized Mathematics, 1(1):181-186, 1990.

[13] Mariusz Żynel and Adam Guzowski. $T_{0}$ topological spaces. Formalized Mathematics, 5(1):75-77, 1996.

Received February 9, 2010 